\title{
Identification of the UDP-glucose-4-epimerase required for galactofuranose biosynthesis and galactose metabolism in A. niger
}

Joohae Park ${ }^{1 \dagger}$, Boris Tefsen ${ }^{2,3+}{ }^{,}$, Mark Arentshorst ${ }^{1 \dagger}$, Ellen Lagendijk$^{1}$, Cees AMJJ van den Hondel ${ }^{1}$, Irma van Die $^{2}$ and Arthur FJ Ram ${ }^{1 *}$

\begin{abstract}
Background: Galactofuranose (Galf)-containing glycoconjugates are important to secure the integrity of the cell wall of filamentous fungi. Mutations that prevent the biosynthesis of Galf-containing molecules compromise cell wall integrity. In response to cell wall weakening, the cell wall integrity (CWI)-pathway is activated to reinforce the strength of the cell wall. Activation of CWI-pathway in Aspergillus niger is characterized by the specific induction of the agsA gene, which encodes a cell wall a-glucan synthase.

Results: In this study, we screened a collection of cell wall mutants with an induced expression of agsA for defects in Galf biosynthesis using a with anti-Galf antibody (L10). From this collection of mutants, we previously identified mutants in the UDP-galactopyranose mutase encoding gene (ugmA). Here, we have identified six additional UDP-galactopyranose mutase (ugmA) mutants and one mutant (named mutant \#41) in an additional complementation group that displayed strongly reduced Galf-levels in the cell wall. By using a whole genome sequencing approach, 21 SNPs in coding regions were identified between mutant \#41 and its parental strain which changed the amino acid sequence of the encoded proteins. One of these mutations was in gene An14g03820, which codes for a putative UDP-glucose-4-epimerase (UgeA). The A to $\mathrm{G}$ mutation in this gene causes an amino acid change of Asn to Asp at position 191 in the UgeA protein. Targeted deletion of ugeA resulted in an even more severe reduction of Galf in N-linked glucans, indicating that the UgeA protein in mutant \#41 is partially active. The ugeA gene is also required for growth on galactose despite the presence of two UgeA homologs in the A. niger genome.
\end{abstract}

Conclusion: By using a classical mutant screen and whole genome sequencing of a new Galf-deficient mutant, the UDP-glucose-4-epimerase gene (ugeA) has been identified. UgeA is required for the biosynthesis of Galf as well as for galactose metabolism in Aspergillus niger.

Keywords: Filamentous fungi, Cell wall, Cell wall integrity, Galactofuranose, Illumina sequencing, Whole genome sequencing, SNP analysis, UDP-glucose-4-epimerase, Mutant screen, Galactose

\section{Background}

The cell wall is an essential component of the fungal cell. Cells can survive the enzymatic removal of the cell wall but the resulting protoplasts need to be stabilized in an environment with high osmolarity to withstand internal turgor pressure. The cell wall of filamentous fungi consists mainly (90\%) of polysaccharide material,

\footnotetext{
* Correspondence: a.f.j.ram@biology.leidenuniv.nl

${ }^{\dagger}$ Equal contributors

'Institute of Biology Leiden, Molecular Microbiology and Biotechnology,

Leiden University, Sylviusweg 72, 2333, BE, Leiden, The Netherlands

Full list of author information is available at the end of the article
}

including polymers of glucose ( $\beta-1,3$ - and $\beta-1,6$-glucans, $\alpha$-1,3-glucan), $\quad N$-acetylglucosamine (chitin), mannose and galactofuranose (galactomannan), galactoaminogalactan and of about $10 \%$ of cell wall glycoproteins (galactomanno-proteins) [1-3]. The different glycoconjugates are either synthesized at the plasma membrane by specific cell membrane-localized synthases (e.g. chitin, $\beta-1,3-$ and $\alpha-1,3$-glucan) or preassembled in the secretory pathway (galactomannan and galactomannoproteins). During or after transport over the membrane, polymers are cross-linked 
with each other via covalent or hydrogen bonds to create a sturdy cell wall.

We and others have previously shown that galactofuranose (Galf) is an important component of the cell wall in Aspergillus species. It is found in several glycoconjugates including galactomannan, secreted and cell wall proteins via $N$ - and $O$-linked chains, and glycosphingolipids (see for review [4]). A key enzyme in Galf biosynthesis is UDPGalp mutase (UgmA), which converts the pyranose form of UDP-galactose (UDP-Gal $p$ ) into UDP-Galf. Only UDPGal $f$ can be transported into the Golgi where Gal $f$ is used as a donor sugar for the synthesis of Galf-containing structures [5-7]. Gene disruption approaches of the ugmA gene in Aspergillus niger, A. fumigatus, A. nidulans have shown that Gal $f$ biosynthesis is required for hyphal morphogenesis and cell wall architecture [8-10], whereas disruption of the ugmA homolog in Cryptococcus neoformans did not have an apparent growth phenotype nor did it affect capsule formation [11]. Inactivation of the ugmA genes in Aspergilli results in an increased sensitivity towards cell wall assembly interfering drugs such as calcofluor white (CFW), indicating that galactofuranosecontaining glycoconjugates are necessary for maintaining the integrity of the cell wall [8].

In $A$. niger the ugmA gene was identified in a screen for mutants in which the cell wall stress reporter gene agsA was constitutively induced [8]. Besides UgmA, proteins required for the biosynthesis of Galf-containing glycoconjugates have been identified in both $A$. nidulans and A. fumigatus. These proteins include UgeA/Uge5, encoding the UDP-glucose-4-epimerase necessary for the synthesis of UDP-Gal $p[12,13]$, UgtA/GlfB encoding a Golgi localized UDP-Galf transporter protein [5,6] and GfsA encoding a Gal $f$ transferase [7].

In this study, we have screened a collection of 240 cell wall mutants with induced agsA expression [8] for mutants that do not secrete Gal $f$ containing glycoconjugates into the growth medium. In addition to a large complementation group of 9 ugmA mutants, one additional Galf-low mutant (\#41), belonging to a different complementation group, was identified. Whole genome sequencing of this mutant revealed that the newly identified Galf-mutant contains a mutation in gene An14g03820. This gene is predicted to encode a putative UDPglucose-4-epimerase gene (ugeA), required for the biosynthesis of Galf as well as for Galp metabolism in Aspergillus niger.

\section{Results}

\section{Screening of Galf-deficient mutants within 240 A. niger} cell wall mutants

To identify additional genes involved in the biosynthesis of cell wall galactomannan in A. niger, we screened a collection of 240 cell wall mutants for Galf-deficient strains. Galf containing structures such as galactomannans and $\mathrm{N}$ - or $\mathrm{O}$-glycosylated proteins are secreted in the medium and therefore analysis of the medium for the presence of Galf using an antibody can identify Gal $f$ minus mutants. From the collection of cell wall mutants, we previously identified the $A$. niger UDP-galactopyranose mutase (UgmA) as an essential protein for the formation of Galf-containing cell wall glycoconjugates ([8], see below). The selection of the three ugmA mutants in that study was not based on their Galf phenotype, but on their Calcofluor white- and SDShypersensitive phenotype. Here, all 240 mutants were grown in liquid medium and $2 \mu \mathrm{l}$ of medium was used in a dot blot analysis using the anti-Gal $f$ antibody L10 [14] as described in Materials and Methods (Figure 1). The screening confirmed the absence of Gal $f$ in the three ugmA mutants already identified (6.13\#44, 15.4\#17, and 6.13\#50) [8], and identified six additional Galf-minus mutants $(15.4 \# 30,15.4 \# 18,15.4 \# 5,15.4 \# 50,15.4 \# 57$ and 6.47\#41). A heterokaryon complementation test using the $\triangle u g m A$ strain was performed to determine whether the newly identified mutants were also mutated in ugmA. In this test, spores of mutants of interest are inoculated close to each other. Outgrowth of the spores will result in the formation of heterokaryotic mycelium in the contact zones. The phenotype of all Galf-deficient mutants identified was characterized by a reduced sporulation (Figure 2A) and this phenotype was used to assay complementation. As shown in Figure $2 \mathrm{~B}$ only mutant \#41 was complemented by the $\triangle u g m A$ mutant, which is visible by a well sporulating zone of heterokaryotic mycelium in the contact zones of the two colonies. The other mutants were not complemented by the $\triangle u g m A$ mutant (data not shown) and therefore considered to be mutant alleles of $u g m A$. In contrast, mutant \#41 showed a clear zone of sporulation in the contact zones with the previously identified $\triangle u g m A$ mutant (Figure 2B) as well as with the $\triangle u g m A$ mutants identified in this study (Figure 2C), indicating that the $\triangle u g m A$ mutant and \#41 mutant are in two different complementation groups. To confirm that the \#41 mutant was not mutated in the ugmA gene, the ugmA locus of the \#41 mutant was amplified by PCR and sequenced. No mutations in the ugmA gene were found.

\section{Mutant \#41 has reduced levels of Galf}

To address the Galf-deficient phenotype of the \#41 mutant further, medium samples of mutant \#41 were analysed using the Platelia assay. This quantitative assay for Galf detection uses the monoclonal EB-A2 antibody which recognizes Galf-moieties on galactomannoproteins [15]. As shown in Figure 3A, titration of the medium samples revealed an approximately 10-fold reduced amount of Galf-reactive epitopes produced by the \#41 mutant compared to that of its parental strain RD6.47. 


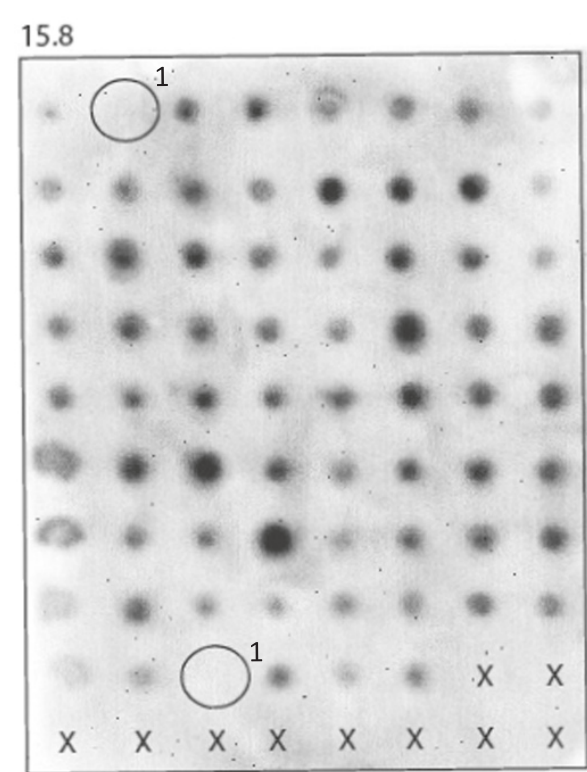

15.4

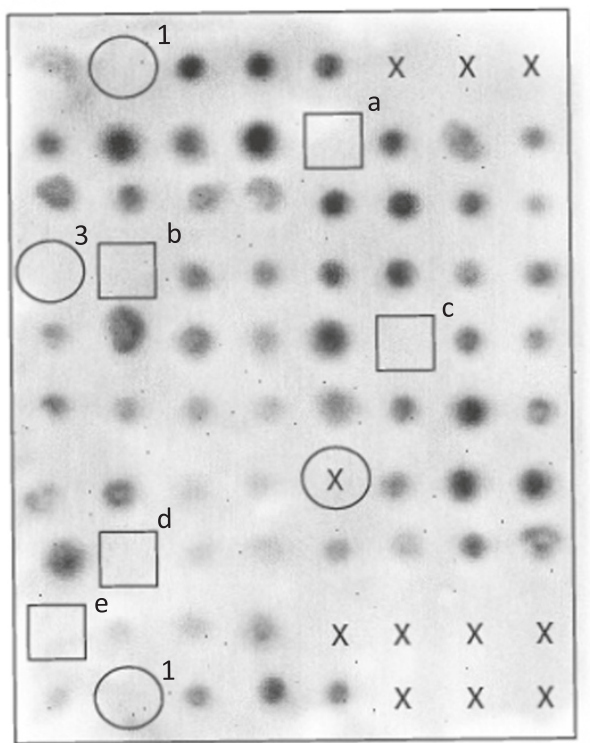

6.13

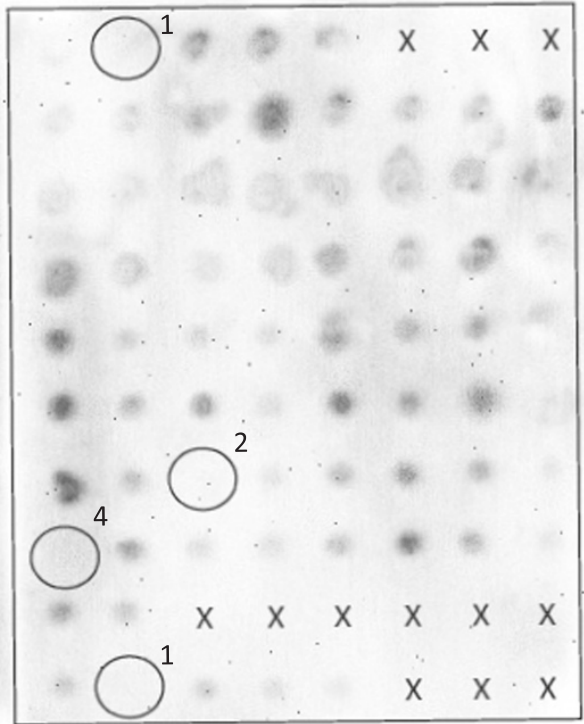

6.47

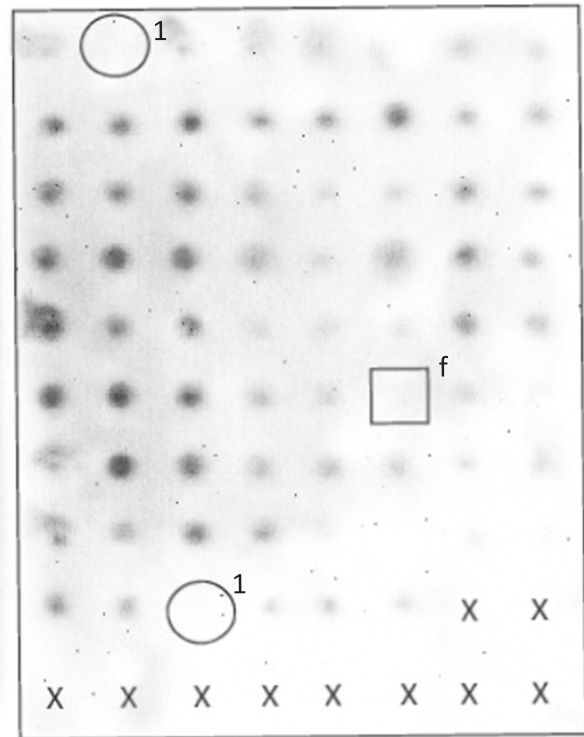

Figure 1 Dot blot assay to detect the presence of Galf residues on secreted glycoconjugates from A. niger mutants. $240 \mathrm{~A}$. niger cell wall mutant strains were grown to early stationary phase and cell-free medium was spotted on nitrocellulose filter paper. The blots were incubated with the anti-Galf antibody (L10) to detect the presence of Galf. Places where no medium was spotted are indicated with X; open circles indicate known Galf-minus mutants ( $1=\Delta$ ugmA, $2=6.13 \# 44 / \mathrm{miaA}, 3=15.4 \# 17 / \mathrm{miaB}, 4=6.13 \# 50 / \mathrm{miaC})$; a mutant that did not grow in this experiment is indicated with $X$ in an open circle; open squares indicate newly detected Galf-minus mutants $(a=15.4 \# 5, b=15.4 \# 18, c=15.4 \# 30, d=15.4 \# 50$, $e=15.4 \# 57$ and $f=6.47 \# 41$ ). $15.8,6.13,15.4$ and 6.47 on top of each blot indicate the different parental backgrounds in which the mutants were made.

As controls, high reactivity with medium from wild type N402 and no reactivity with medium from the $\Delta u g m A$ strain were measured in this assay. Western blot analysis of extracellular medium proteins using the L10 antibody also detected less Galf epitopes on secreted glycoproteins in contrast to the wild type strains $\mathrm{N} 402$ and RD6.47, but clearly more than in the medium of the $\triangle$ ugmA strain (Figure 3B). Detection of $N$-glycans with the lectin ConcavalinA (ConA) was performed as a control.
To obtain more insight into the presence of Gal $f$ in the cell wall of the \#41 mutant, galactomannan from two Galf mutants ( $\triangle u g m A$ and \#41), and the wild-type strain (N402) was isolated essentially as described previously by Bardalaye and Nordin, 1977 [16] (see Methods for details). A titration of the purified galactomannan fraction was applied to the Platelia assay, revealing again a lowered reactivity of the Galf-antibody with the galactomannan derived from \#41 compared to the wild-type strain (Figure 3C), 


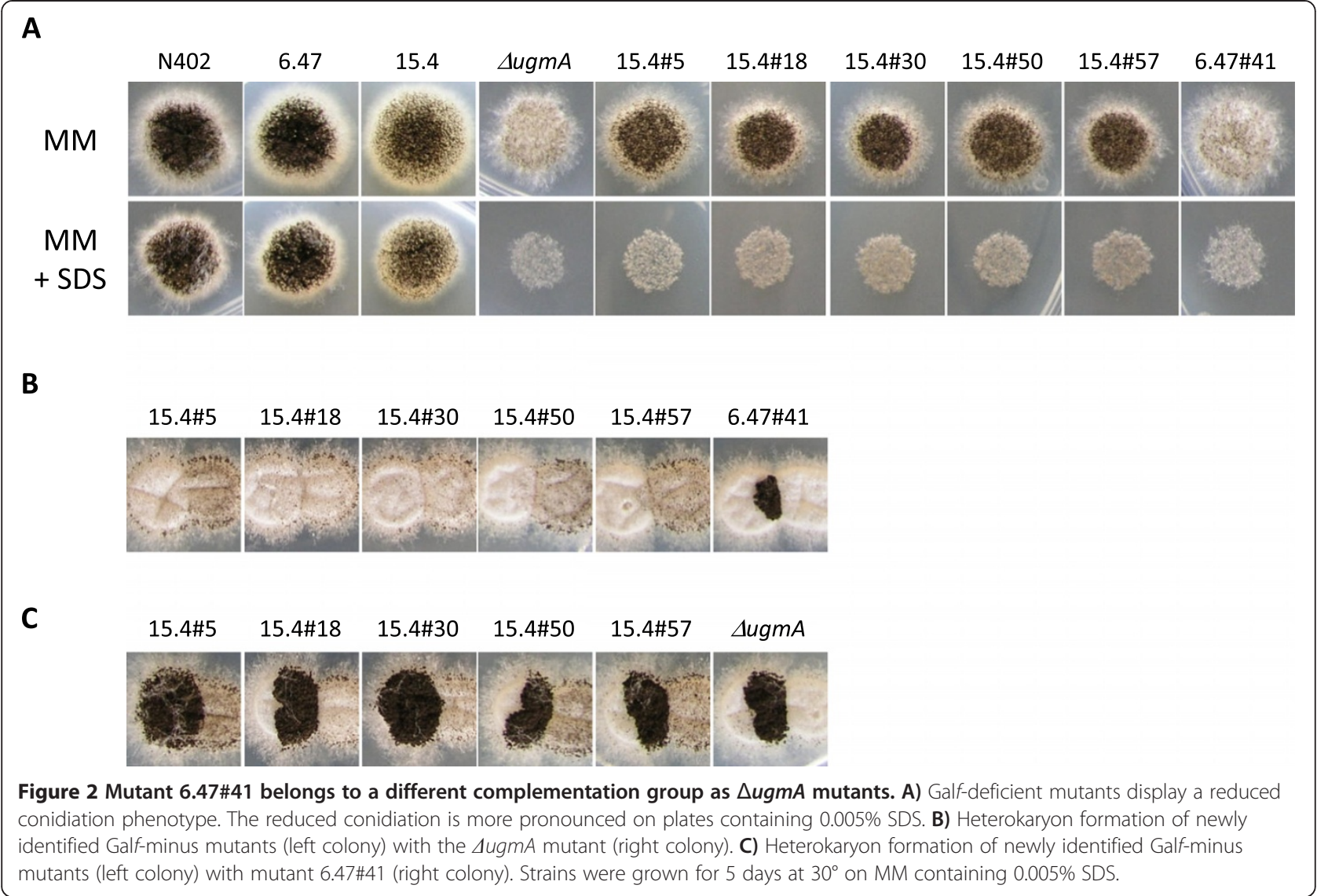

whereas the polysaccharide isolated from $\triangle u g m A$ was not reactive at all. This indicated that there are less Galf-moieties present on the galactomannan from \#41 compared to wild type galactomannan. To confirm this result, the monosaccharide composition of the galactomannan fraction of these strains was analysed by HPAEC analysis (DIONEX system) after hydrolysis (Figure 3D). As expected, no Galf $f$ was detected in the hydrolyzed polysaccharide isolated from the $\Delta u g m A$ mutant. The ratio Galf /Man of the wild-type strains was found to be well above 2 (2.2-2.8), while this ratio was only 0.5 for the \#41 mutant. These results demonstrate that the amount of Gal $f$ is reduced more than 4-fold in the \#41 mutant compared to its parent strain. This lowered amount of Gal $f$ apparently has escaped detection by the L10 antibody in our initial screening by dot blot, leading to its discovery (Figure 1), but clearly is detected by the Platelia assay (Figure 3A and $3 \mathrm{C})$, using the EB-A2 antibody, as well as by Western blot analysis using the L10 antibody (Figure 3B).

\section{Genomic characterization of mutant \#41 by whole genome sequencing}

An A. niger genomic cosmid library [17] was used in several unsuccessful attempts to complement the reduced sporulating growth phenotype of mutant \#41. As an alternative approach, the genome of both the parental strain (RD6.47) and the \#41 mutant were sequenced by pair-end Illumina sequencing. A single nucleotide polymorphism (SNP) analysis between the two strains was performed and 78 SNPs were identified (Additional file 1: Table S1). These SNPs were analysed further by determining whether a particular SNP was located in a predicted ORF and whether this SNP affected the amino acid sequence. We identified 21 SNPs in genes in the \#41 mutant that caused changes in the amino acid sequence (Additional file 2: Table S2). Most of these SNPs seemed unrelated to Galf-biosynthesis, but the mutation in gene An14g03280 encoding a putative UDP-glucose-4-epimerase seemed important as, it was recently published that the UDP-glucose4-epimerase (UgeA) is needed for Galf biosynthesis in $A$. nidulans [13]. The mutation in the ugeA (An14g03280) gene of mutant \#41 (A to $G$ ) caused the change of a codon from $\mathrm{AAC}$ to $\mathrm{GAC}$ which consequently resulted in the change of Asn to Asp at position 191 in the UgeA protein. The mutation identified by the Illumina genome sequencing approach was confirmed by PCR amplification of the ugeA locus of the \#41 strain followed by direct sequencing. 

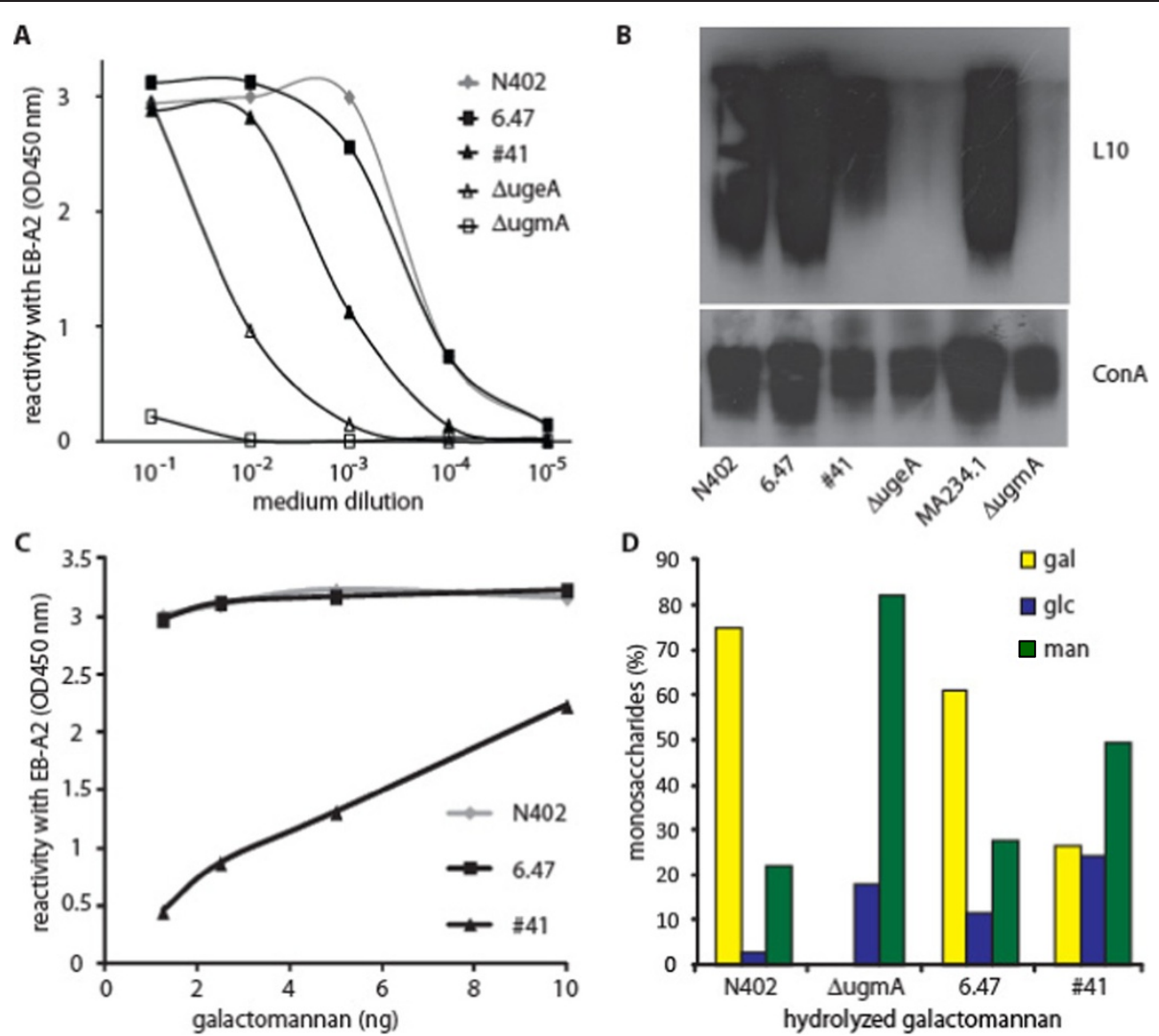

Figure 3 Galf in mutant \#41 is greatly reduced but not absent. A) Platelia assay with anti-Galf antibody EB-A2 to detect Galf residues in a dilution range of medium samples from the indicated strains B) Western blot analysis with anti-Galf antibody L10 on medium samples from the indicated strains (upper blot) and ConA lectin (lower blot) C) Platelia assay on purified galactomannan to analyse the presence of Galf $\mathbf{D}$ ) Galactomannan from the indicated strains was hydrolyzed and their sugar composition was analyzed by HPAEC and depicted here as a percentage of the total amount of sugar in each sample.

\section{Disruption of the A. niger ugeA gene and phenotypic analysis}

To confirm that the mutation in An14g03820 (ugeA) caused the Galf-low phenotype in mutant \#41, a pyrG based gene deletion cassette was made. The deletion cassette ( $p \Delta u g e A:: p y r G$ ) was transformed to MA169.4 and uridine prototrophic transformants were purified. During purification of the primary transformants it was found that most of the transformants displayed a reduced growth and a reduced sporulation phenotype, which resembled the phenotype of the \#41 mutant. Subsequent diagnostic PCR and Southern blot analysis proved that the transformants with the growth phenotype contained an ugeA deletion (data not shown). Further phenotypic characterization of the $\Delta u g e A$ mutant showed similar morphological alterations (increased branching and irregular length of hyphal compartments) as found for the \#41 mutant (data not shown).

As demonstrated above, very low levels of Gal $f$ were observed in the dot blot (Figure 1) and Platelia assay (Figure 3A) for the \#41 mutant. Interestingly, the amount of Galf in the $\Delta u g e A$ strain was strongly reduced compared to the \#41 mutant, indicated by the complete lack of signal on the dot blot (Figure 4) and a further 10 -fold reduction of the Galf-signal in the Platelia assay (Figure 3A). These results suggest that the UgeA protein in \#41 is still slightly active and able to convert some UDP-Glc $p$ to UDP-Gal $p$, which is subsequently utilized for Galf-biosynthesis. Despite the observation that more Galf is present in the \#41 mutant compared to the $\Delta u g e A$ mutant, both mutants display similar phenotypes, although some subtle differences could be seen between the \#41 and the $\Delta u g e A$ mutants. The \#41 mutant seems to sporulate slightly more intense on the MM plate and grows somewhat better on the $0.005 \%$ SDS plate (Figure 5).

To further confirm that the mutation in ORF An14g03820 (ugeA) in the \#41 mutant and the deletion of ugeA are the cause of the observed phenotypes, a complementation analysis was performed. Therefore, $p y r G^{-}$derivatives were obtained from the \#41 and $\triangle u g e A$ mutants by selecting on 5 'FOA plates. The ugeA gene was PCR amplified and cloned into an autonomously replicating vector (pAMA-pyrG, [18]) and used for complementation. Transformation of the pAMA-ugeA plasmid to the \#41 and $\triangle u g e A$ mutants 


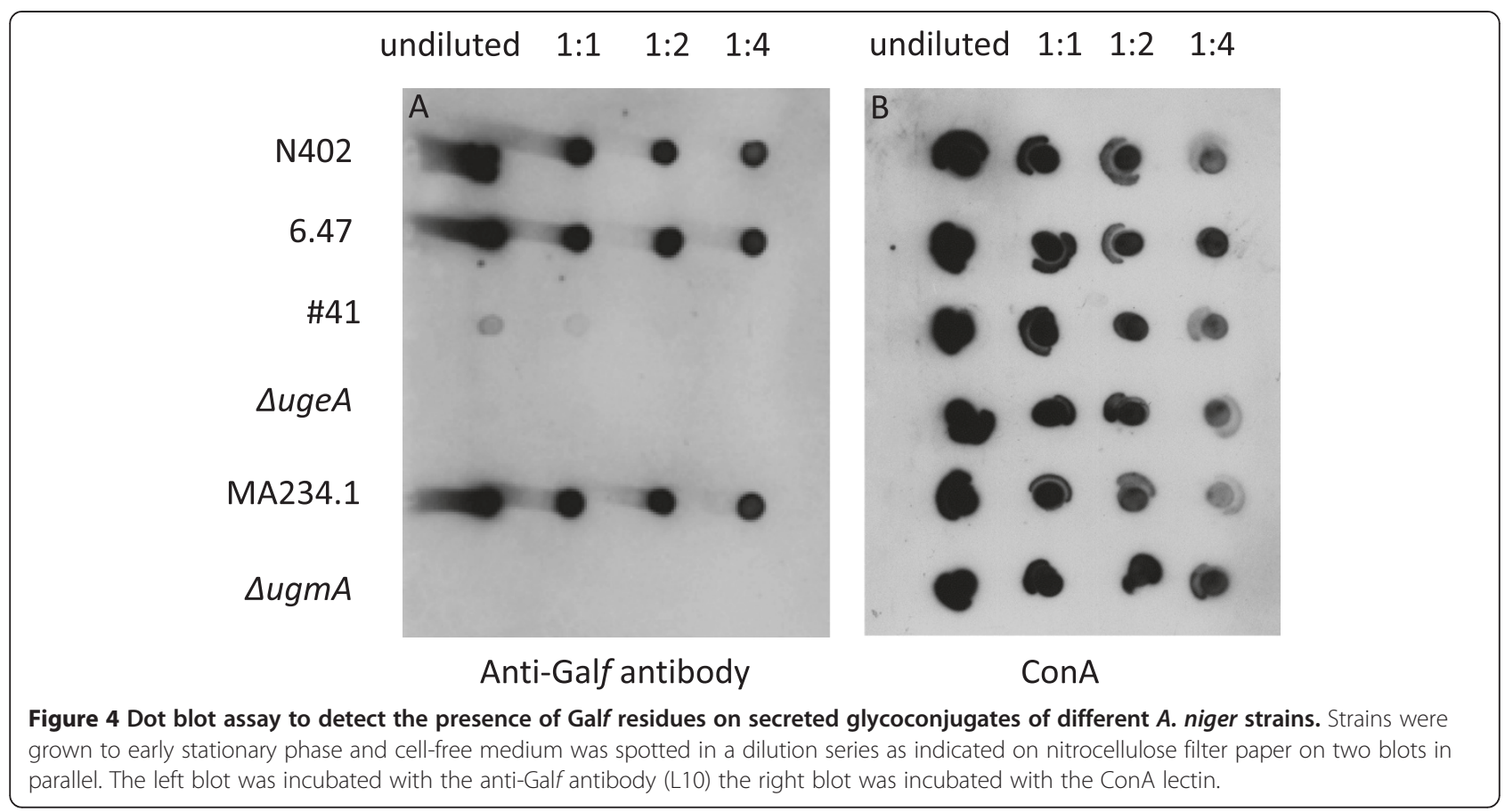

complemented the reduced growth and sporulation phenotype (Figure 6A and B) as well as the Galf-negative phenotype in the dot blot analysis (data not shown). Control transformations with the empty plasmid (pAMApyrG) or the pAMA-ugmA-pyrG gene were also performed. In general, the transformation of these control plasmids did not complement the sporulation defect of the \#41 and $\Delta u g e A$ mutants. Unexpectedly, we occasionally (with a frequency of $0.2 \%-1 \%$ ) obtained transformants with the empty or ugmA plasmid that sporulated well and grew like the complemented strain. From the original transformation plates of the $\Delta u g e A$ mutant with the pAMA-pyrG or the pAMA-ugmA-pyrG plasmids, two transformants were purified with the improved sporulation phenotype. The pAMA-pyrG or pAMAugmA-pyrG transformant that sporulated well were also Galf-positive in the dot blot analysis (data not shown).
The reversion of the phenotype might be caused by second site suppressor mutations or by increased expression of redundant genes (see Discussion).

\section{UgeA is required for growth on galactose in $A$. niger}

Based on sequence comparisons, UgeA (An14g03820) is predicted to encode a UDP glucose-4-epimerase and the result above clearly indicate that this gene is most likely responsible for the synthesis of UDP-Gal $p$, which is further converted to UDP-Galf by the UgmA enzyme. $A$. niger contains in addition to UgeA (An14g03820) two other homologous genes predicted to encode UDP glucose-4epimerases (An12g10410 and An02g11320). The presence of three putative UDP glucose-4-epimerases in $A$. niger is similar to A. fumigatus in which the function of the three genes (uge3, uge5 and uge4) was recently analysed [12]. Based on homology, the A. niger UgeA is most similar to

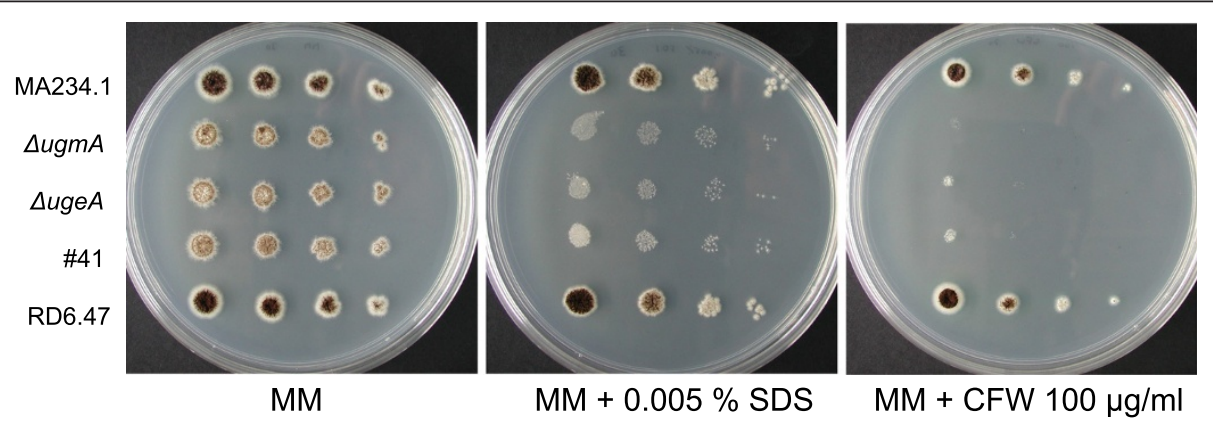

Figure 5 Susceptibility of $\boldsymbol{A}$. niger Galf mutants towards SDS and CFW. Ten-fold serial dilutions of spores derived from different Galf mutants were spotted on minimal medium plates containing $0.005 \%$ SDS or $100 \mu \mathrm{g} / \mathrm{ml} \mathrm{CFW}$. Pictures were taken after three days of growth at $30^{\circ} \mathrm{C}$. 


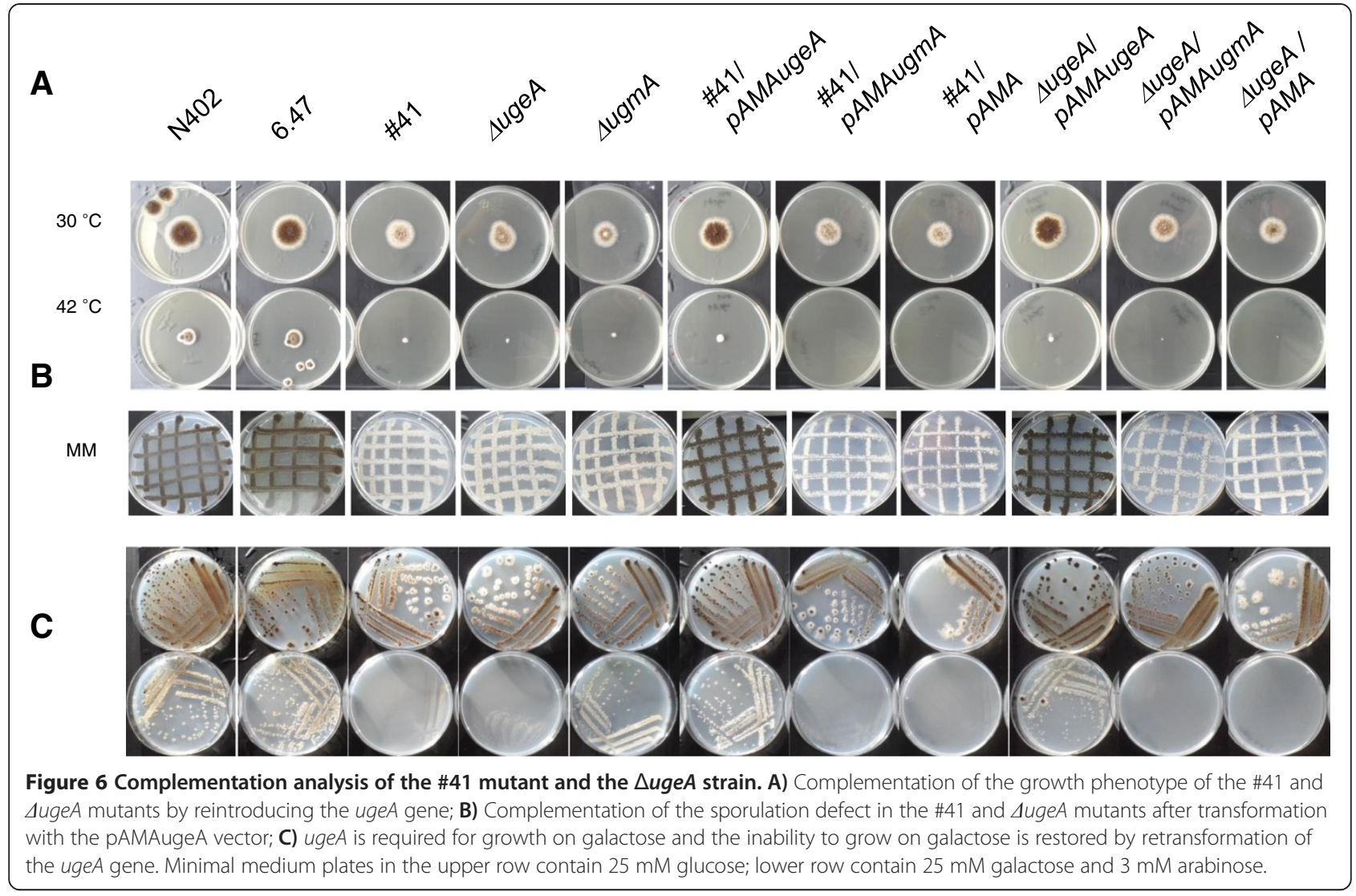

the A. fumigatus Uge5 protein and the UgeA protein of $A$. nidulans (See Table 1 for amino acid identities of the Uge homologs between the three species). To test whether the $A$. niger ugeA gene is required for growth on galactose, mutant \#41, $\triangle u g e A$ and their respective parental wild type strains were inoculated on plates containing $50 \mathrm{mM}$ galactose and $0.3 \mathrm{mM}$ arabinose. The supplementation of a second carbon source in addition to galactose is included because $A$. niger spores are unable to germinate on galactose alone [19-21]. As shown in Figure 6C, the \#41 and $\triangle u g e A$ mutants do not grow on galactose medium indicating that ugeA is required for growth on galactose. The inability to grow on galactose of the ugeA strain is not a consequence of its inability to produce Galf as the ugmA deletion strain grows normally on galactose (Figure 6C).

\section{Discussion}

Screening of a collection of $A$. niger cell wall mutants, which were previously isolated by induced expression of the cell wall stress reporter gene (ags $A)$, identified in

Table 1 Comparison of the percentage of amino acid identity between Uge proteins of $A$. niger, A. fumigatus, and A. nidulans

\begin{tabular}{|c|c|c|c|c|c|c|c|c|c|}
\hline \multirow[t]{2}{*}{ Species } & \multirow[t]{2}{*}{ Protein ID } & \multicolumn{3}{|l|}{ A. niger } & \multicolumn{3}{|l|}{ A. fumigatus } & \multicolumn{2}{|l|}{ A. nidulans } \\
\hline & & $\begin{array}{l}\text { UgeA } \\
\text { An14g03820 }\end{array}$ & $\begin{array}{l}\text { UgeB } \\
\text { An12g10410 }\end{array}$ & $\begin{array}{l}\text { UgeC } \\
\text { An02g11320 }\end{array}$ & $\begin{array}{l}\text { Uge3 } \\
\text { Afu3g07910 }\end{array}$ & $\begin{array}{l}\text { Uge4 } \\
\text { Afu4g14090 }\end{array}$ & $\begin{array}{l}\text { Uge5 } \\
\text { Afu5g10780 }\end{array}$ & $\begin{array}{l}\text { UgeA } \\
\text { AN4727.2 }\end{array}$ & $\begin{array}{l}\text { UgeB } \\
\text { AN2951.2 }\end{array}$ \\
\hline \multirow[t]{3}{*}{ A. niger } & UgeA (An14g03820) & $100^{*}$ & 40 & 39 & 39 & 37 & 94 & 94 & 39 \\
\hline & UgeB (An12g10410) & & 100 & 66 & 58 & 59 & 41 & 39 & 58 \\
\hline & UgeC (An02g11320) & & & 100 & 86 & 59 & 40 & 40 & 81 \\
\hline \multirow[t]{3}{*}{ A. fumigatus } & Uge3 (Afu3g07910) & & & & 100 & 58 & 39 & 39 & 84 \\
\hline & Uge4 (Afu4g14090) & & & & & 100 & 37 & 39 & 56 \\
\hline & Uge5 (Afu5g10780) & & & & & & 100 & 94 & 37 \\
\hline \multirow[t]{2}{*}{ A. nidulans } & UgeA (AN4727.2) & & & & & & & 100 & 39 \\
\hline & UgeB (AN2951.2) & & & & & & & & 100 \\
\hline
\end{tabular}

* values indicate percentage of amino acids identities after pairwise alignment using BLASTP at http://blast.ncbi.nlm.nih.gov/Blast.cgi. 
total 9 mutants which did not secrete detectable levels of Galf-containing glycoconjugates into the medium. Three mutants were identified previously [8] and six new Galfdeficient mutants were additionally found in this screen. Interestingly, the mutants fell into two unevenly distributed complementation groups. A large group (represented by eight alleles) consisted of mutants in the ugmA gene and the other complementation group only contained a single representative with a mutation in the uge $A$ gene. The reason for this uneven distribution among the two complementation groups is not clear as both the ugmA and the ugeA mutants have very similar phenotypes when inactivated (Figures 4 and 5). Besides UgeA and UgmA, which are required for the synthesis of the UDP-Galf, proteins involved in subsequent steps of the synthesis of Galfcontaining glycoconjugates have been recently identified and include a specific UDP-Gal $f$ transporter (GlfB in $A$. fumigatus [5] and UgtA in A. nidulans [6] and a Galftransferase [7]. Mutants in the homologs of those genes in $A$. niger were not identified in our cell wall mutant collection. Analysis of the $A$. niger genome showed the presence of two homologs of GlfB/UgtA and three homologs of the GfsA protein (data not shown). The inability to detect representative mutants in the cell wall mutant collection suggests possible redundant functions of the pairs of genes in $A$. niger. Indeed, the two $A$. niger Galf transporters only display a Galf minus phenotype when both genes were deleted (Park and Ram, unpublished data).

This study represents the first example of the power of genome sequencing for characterizing classically obtained mutants in $A$. niger. The mutant \#41 strain was created by UV mutagenesis which is known to preferably induce transitions (purine to purine (A-G) or pyrimidine to pyrimidine (T-C) mutations. From the 78 SNPs a majority of 49 (63\%) SNPs were transitions and 29 (27\%) were found to be transversions (purine to pyrimidine or reverse) (Additional file 1: Table S1). Of the 29 transversions, a high number of $\mathrm{A}$ to $\mathrm{T}$ transversions were found. The total number of mutations (21) in non-exonic regions was relative low taking into account the relative stringent dosis of UV treatment (66\% survival). This number of SNPs makes it feasible to identify responsible mutants in the case that analysis of the predicted function of the mutated genes does not give a clear clue.

Interestingly, the Asn to Asp mutation at position 191 in UgeA demonstrated in mutant \#41 seems to inhibit enzyme function greatly, but not completely. We aligned the amino acid sequences of UgeA from $A$. niger with that from $A$. nidulans, human and Escherichia coli (Additional file 3: Figure S1A). This shows that the Asn at position 191 is conserved between the Aspergilli and human, but not in E. coli. Recently, the complex crystal structure of UgeA from $A$. nidulans together with its substrates NAD + and UDP-Glcp was elucidated [22], which showed a similar orientation as in the human enzyme (GALE). This structure enabled us to visualize the location of Asn191 at the start of $\beta$-strand 6 (in red, Additional file 3: Figure S1B). Mutation of this residue to an Asp will result in the disruption of the ideal orientation of $\beta$-strands 6 and 7, and thereby of the substrateorientation YFN-domain (residues 197-199). Apparently, this disruption is enough to diminish the UgeA enzyme activity greatly, resulting in lower amounts of available Galp to flow into the Galf-biosynthesis pathway.

This study shows that the uge $A$ gene of $A$. niger is required for UDP-Gal $f$ biosynthesis and for growth on galactose. This is identical to the situation in A. nidulans in which it has been shown that the $\Delta u g e A$ mutant also lacks Gal $f$ and is unable to grow on galactose [13]. Sequence comparison showed that the $A$. nidulans UgeA protein is most similar to the UgeA protein of $A$. niger (Table 1 ). In $A$. fumigatus, the uge 5 mutant (Uge5 is the paralog of both $A$. niger and $A$. nidulans UgeA protein) can grow on galactose medium. It has recently been shown that the A. fumigatus Uge3 protein is responsible for the growth on galactose as disruption of both uge 3 and uge 5 results in a galactose auxotrofic strain [12]. The galactose auxotrophy of the $A$. niger $\triangle u g e A$ and $A$. nidulans $\Delta u g e A$ mutants either indicated that the Uge3 homologs which are present in the genome are not expressed or have a more specific activity. The Uge3 protein of A. fumigatus has been shown to be involved in galactosaminogalactan synthesis and can mediate both the production of UDP-galactose and UDP$N$-acetyl-galactosamine. Further enzymatic and molecular genetic analysis of the Uge homologs in $A$. niger and $A$. nidulans is required to understand their exact function and their possible role in galactosaminogalactan biosynthesis. In this respect, it is interesting that we observed high frequency of suppression of the $\Delta u g e A$ phenotype after transformation. Although the phenotype of the \#41 and $\triangle u g e A$ mutants were stable during normal propagation, transformation of both strains yielded regularly suppressor mutations that were restored in their ability to produce Galf-decorated galactomannoproteins and were also restored in their ability to grow on galactose (data not shown). A possible mechanism to explain the development of suppressors include activation of expression of one of the uge genes, or occurrence of a mutation in one of these genes that changes substrate specificity.

\section{Conclusion}

By screening a collection of UV-generated cell wall mutants, a new Galf-deficient mutant was identified. We used whole genome sequencing to identify the mutation responsible for this mutant phenotype. Complementation and targeted deletion studies confirmed that the UDP-glucose-4-epimerase gene (ugeA) is required for 
the biosynthesis of Gal $f$ as well as for galactose metabolism in Aspergillus niger.

\section{Methods}

\section{Strains and culture conditions}

The Aspergillus niger strains used in this study are listed in Table 2. Strains were grown on minimal medium (MM) [23] containing $1 \%$ (wv-1) glucose as carbon source or complete medium $(\mathrm{CM})$ containing $0.5 \%(\mathrm{wv}-1)$ yeast extract, $0.1 \%$ (wv-1) casamino acids in addition to MM. When required, plates were supplemented with $10 \mathrm{mM}$ uridine. $5^{\prime}$ FOA selection for the selection of $p y r G^{-}$strains was performed as described previously [24]. For the plate growth assays, strains were grown on MM plates containing $25 \mathrm{mM}$ glucose, $25 \mathrm{mM}$ galactose, or $25 \mathrm{mM}$ galactose and $3 \mathrm{mM}$ arabinose. For the heterokaryon assay, MMplates were supplemented with $0.005 \%$ SDS.

\section{Screening methods to identify Galf minus mutants}

Strains from the collection of 240 cell wall mutants of $A$. niger [8] were grown in $25 \mathrm{ml} \mathrm{CM}$ in $50 \mathrm{ml}$ tube Greiner tube for $24 \mathrm{~h}$ at $30^{\circ} \mathrm{C}$. Medium samples were filtered over a Whatman glass microfiber filter and $2 \mu \mathrm{l}$ medium was spotted on nitrocellulose blotting paper. Blots were incubated with the L10 monoclonal anti-Galf-antibody (1:10) in TSMT (TSM [20 mM Tris-HCl, pH 7.4, $150 \mathrm{mM} \mathrm{NaCl}, 2 \mathrm{mM} \mathrm{CaCl}_{2}, 2 \mathrm{mM} \mathrm{MgCl}$ ] with $0.05 \%$ Tween-20) supplemented with 5\% BSA or with peroxidase labeled ConA (ConA-PO EY Laboratories, USA) used in a 1:500 dilution in PBS containing $0.05 \%(\mathrm{v} / \mathrm{v})$

Table 2 Aspergillus niger strains used in this study

\begin{tabular}{|c|c|c|}
\hline Strain & Description & References \\
\hline N402 & cspA1 derivative of ATCC9029 & Bos et al. 1988 \\
\hline MA70.15 & $\Delta k u s A:: a m d S, p y r G-$ & [25] \\
\hline MA169.4 & $\Delta k u S A:: D R \_a m d S \_D R, p y r G-$ & [18] \\
\hline MA234.1 & MA169.4 transformed with pAB4.1 to make strain pyrG+ & Arentshorst, unpublished \\
\hline RD6.47 & pPagsA-amdS-TamdS-pyrG* and pPagsA-H2B-GFP-TtrpC/pAN7.1 & [8] \\
\hline RD6.47\#41 & galF- mutant derived from RD6.47 & [8] \\
\hline RD6.47\#41, pyrG- & pryG- derivative of RD6.47\#41 & This study \\
\hline RD6.47\#41/pAMA_ugeA & RD6.47\#41/pyrG-pBlue_AMA_ugeA & This study \\
\hline RD6.47\#41/pAMA_ugmA & RD6.47\#41/pyrG-pBlue_AMA_ugmA & This study \\
\hline RD6.47\#41/pAMA & RD6.47\#41/pyrG-pBlue_AMA & This study \\
\hline JH12.1 & ugeA (An14g03820)::pyrG deletion in MA169.4 & This study \\
\hline $\mathrm{JH} 21.1$ & pyrG- derivative of $\mathrm{JH} 12.1$ & This study \\
\hline$J H 21.1 .1$ & $\Delta u g e A / p A M A \_u g e A$ & This study \\
\hline$J H 21.1 .2$ & $\Delta u g e A / p A M A \_u g m A$ & This study \\
\hline$J H 21.1 .3$ & $\Delta u g e A / p A M A$ & This study \\
\hline MA87.6 & ugmA (An02g08660)::pyrG in MA70.15 & [8] \\
\hline MA247.2.1b & pryG- derivative of MA87.6 & This study \\
\hline MA247.2.1b.1 & $\Delta u g m A / p A M A \_u g e A$ & This study \\
\hline MA247.2.1b.2 & $\Delta u g m A / p A M A \_u g m A$ & This study \\
\hline MA247.2.1b.3 & $\Delta u g m A / p A M A$ & This study \\
\hline RD15.4 & pPagsA-H2B-GFP-TtrpC-pyrG* and pPagsA-amdS-TamdS/pAN7.1 & [8] \\
\hline RD15.4\#5 & galf- mutant derived from RD15.4 & [8] \\
\hline RD15.4\#18 & galF- mutant derived from RD15.4 & [8] \\
\hline RD15.4\#30 & galf- mutant derived from RD15.4 & [8] \\
\hline RD15.4\#50 & galf- mutant derived from RD15.4 & {$[8]$} \\
\hline RD15.4\#57 & galF- mutant derived from RD15.4 & [8] \\
\hline RD6.13\#44 (miaA) & ugmA mutant derived from RD6.13 & [8] \\
\hline RD15.4\#17 (miaB) & ugmA mutant derived from RD15.4 & [8] \\
\hline RD6.13\#50 (miaC) & ugmA mutant derived from RD6.13 & {$[8]$} \\
\hline
\end{tabular}

pyrG+ = pyrG plus (uridine prototroph); $p y r G-=$ pyrG minus (uridine auxotroph); $p y r G^{*}=$ mutant pyrG allele for targeting at the pyrG locus). 
Tween-20. Blots with the L10 antibody were subsequently incubated with rabbit-a-mouse-HRP (PO161, DAKO) as a secondary antibody in TSMT with 5\% BSA. For detection of the secondary antibody or the ConA-PO conjugate, a chemiluminescence kit (Thermo Scientific Pierce) was used.

\section{Isolation of cell wall galactomannan}

Cell walls were isolated from $A$. niger wild type strain N402 and the \#41 mutant strain after growth in CM for $24 \mathrm{~h}$ at $30^{\circ} \mathrm{C}$ at $250 \mathrm{rpm}$. Spores $\left(1 \times 10^{9}\right)$ were inoculated in $1 \mathrm{~L}$ of $\mathrm{CM}$ in a $2 \mathrm{~L}$ Erlenmeyer. The mycelium of both strains was isolated by filtering over myracloth and grinded in liquid nitrogen using a pestle and mortar and the broken mycelia washed 3 times with $1 \mathrm{M} \mathrm{NaCl}$ and 3 times with MilliQ at $4^{\circ} \mathrm{C}$ by centrifugation $(3600 \mathrm{rpm}$, $10 \mathrm{~min})$. Successful breakage of the mycelia was confirmed by microscopy. Isolated cell walls were lyophilized for 24 h using 1.0 gram of cell walls (dry weight) for both the N402 and the \#41 strain. The galactomannan fraction was isolated according to Bardalaye and Nordin [16]. The yield of purified galactomannan was $21.2 \mathrm{mg}$ and $10.4 \mathrm{mg}$ from 1 gram of freeze dried cell walls for the N402 and \#41 strain, respectively.

\section{Platelia assay}

Microtiter plate wells containing coated antibody EB-A2, which recognizes Galf-moieties on galactomannan [14] were filled with $50 \mu \mathrm{l}$ EB-A2 conjugated to HRP (all from Platelia Aspergillus EIA kit, Bio-Rad). Supernatant of $A$. niger strains or purified galactomannan was added to the wells and incubated for $90 \mathrm{~min}$ at $37^{\circ} \mathrm{C}$. After washing 5 times, $200 \mu \mathrm{l}$ 3,3',5,5' -tetramethylbenzidine (TMB) detection mixture was added and samples were incubated for $30 \mathrm{~min}$ at ambient temperature in the dark. After stopping the coloring reaction by addition of $100 \mu \mathrm{l} 1.5 \mathrm{~N} \mathrm{H}_{2} \mathrm{SO}_{4}$, relative amounts of Galf on galactomannan were determined by measuring the optical density (OD) by spectrophotometry at $450 \mathrm{~nm}$.

\section{Monosaccharide analysis by HPAEC}

Galactomannan isolated from $A$. niger was analyzed for its monosaccharide composition by High-Performance Anion Exchange Chromatography (HPAEC), using the method described by [26] with minor modifications. In summary, chromatography of the samples was performed in a Dionex, Bio-LC system, using a CarboPac PA 10 column $(250 \times 2 \mathrm{~mm})$ in combination with a CarboPac guard column $(2 \times 50 \mathrm{~mm})$, Dionex Corp. Around $2 \mathrm{mg}$ galactomannan was dissolved in $2 \mathrm{ml}$ water and incubated with $360 \mu \mathrm{l}$ of TFA for $5 \mathrm{~h}$ at $100^{\circ} \mathrm{C}$. Hereafter, $3 \mathrm{ml} \mathrm{MilliQ}$ water was added and samples were freeze dried overnight. The pellets were dissolved in $100 \mu \mathrm{l}$ water. After diluting 1:100, $20 \mu \mathrm{l}$ (equivalent to $4 \mu \mathrm{g}$ polysaccharide) was injected onto the column. Monosaccharides were eluted isocratically using $\mathrm{H}_{2} \mathrm{O}$ and $1 \mathrm{M} \mathrm{NaOH}$ in a ratio of $80: 20$ for $30 \mathrm{~min}$. The column was washed with water for $10 \mathrm{~min}$ and equilibrated with $250 \mathrm{mM} \mathrm{NaOH}$ for $10 \mathrm{~min}$ before every injection.

\section{Western blot analysis}

Medium samples of the various strains were obtained as described for the dot-blot analysis and $20 \mathrm{ul}$ of culture filtrate was used for Western blot analysis. SDS-PAGE and blotting were carried out as described [27]. For labeling with the anti-Galf antibody (L10), the membrane was blocked with 5\% low-fat milk in TTBS (Tris-buffered saline, 0.05\% Tween-20), and Galf was detected using the L10 antibody $(1 / 5,000)$ overnight, followed by a goat anti-rabbit horseradish peroxidase secondary antibody $(1 / 20,000)$ for $1 \mathrm{~h}$. Detection was performed using a chemiluminescence kit (Thermo Scientific Pierce), according to manufacturer's instructions.

\section{Genetic methods}

Fungal transformations were done according the protoplast method described by [24]. Complementation tests were done using the heterokaryon test. 2 ul of spores $\left(1 \times 10^{6}\right.$ spores $\left./ \mathrm{ml}\right)$ of strains to be tested were inoculated on MM plates containing $0.005 \%$ SDS approximately $1 \mathrm{~cm}$ apart from each other. Plates were incubated for 3 days at $30^{\circ} \mathrm{C}$ and analyzed for a zone of conidiation of heterokaryotic mycelium.

\section{Plasmid construction}

For the construction of the ugeA deletion cassette the Multisite GatewayR Three-Fragment Vector Construction kit was used. As a marker for deleting the ugeA gene, the pyrG marker of $A$. oryzae was used. To facilitate removal of the AopyrG marker, $A$. nidulans tTrpC repeats were cloned around the pyrG gene [28]. The TrpC-pyrGTrpC fragment was PCR amplified using primer listed in Table 3 and cloned in pDonor221. The $5^{\prime}$ and $3^{\prime}$ flanking regions of ugeA were PCR amplified using primers with appropriate attB sites (Table 3 ) and the 799 bp fragments were cloned into pDONR P4-P1R and pDONR P2R-P3 respectively. The subsequent LR reaction was performed using pDONR_ugeA5, pDONR_ugeA3 and pDONR_TrpC_pyrG_TrpC and pDEST R4-R3 Vector 2 to create the $u g e A$ deletion plasmid. The final construct was verified by restriction analysis and sequencing.

For the complementation analysis the autonomously replicating vector pMA172 was used [18]. The ugeA or $u g m A$ genes including promoter and terminator regions were PCR amplified using primers listed in Table 3, cloned into pJet2.1 and verified by sequencing. The uge $A$ and $u g m A$ genes were reisolated as $\operatorname{SmaI}(u g e A)$ or Not $\mathrm{I}$ $(u g m A)$ fragments and cloned into the unique $S m a \mathrm{I}$ or 
Table 3 The primers used in this study

\begin{tabular}{lll}
\hline Name & Sequence 5'-3' & Aplication \\
\hline attB1_TrpC_CFP_F & ggggacaagtttgtacaaaaaagcaggctATGGACGAGCTGTACAAGTAA & Amplification TrpC-pyrG-TrpC cassette \\
attB2r_TrpC_R & ggggaccactttgtacaagaaagctgggtTGGGTGTACGGAGCATTCACTAGGC & Amplification TrpC-pyrG-TrpC cassette \\
attB4_ugeA5F & ggggacaactttgtatagaaaagttgCCGATAGGAAGGATGAGGAT & Amplification 5'flank ugeA disruption cassette \\
attB1r_ugeA5R & ggggactgcttttttgtacaaacttgGATGATGATAAGGTATGACT & Amplification 5'flank ugeA disruption cassette \\
attB2r_ugeA3F & ggggacagctttcttgtacaaagtggATAATGACCCCGCATATGTT & Amplification 3'flank ugeA disruption cassette \\
attB3_ugeA3R & ggggacaactttgtataataaagttgGGATGGACAGCCGTGCAGTG & Amplification 3'flank ugeA disruption cassette \\
ugmAP1f-Notl & aaggaaaaaagcggccgcAGGACTCCATAGGCCCGTAGA & Amplification ugmA for complementation and sequencing \\
ugmAP2r-Notl & aaggaaaaaagcggccgcAGAAACGGACTGCATGGGC & Amplification ugmA for complementation and sequencing \\
ugeAfw-Smal & ctcgagcccgggTTGATTGGACCCTTGGGATCG & Amplification ugeA for complementation and sequencing \\
ugeArev-Smal & ctcgagcccgggTTGGCAAGGAAGGAGGTGAAG & Amplification ugeA for complementation and sequencing \\
\hline
\end{tabular}

Italic letters indicate attB sites, restriction sites are shown in bold.

NotI site of pMA172 to give pAMA-ugeA and pAMAugmA respectively.

\section{Genome sequencing and SNP identification}

Genomic DNA isolations of strains 6.47 (parental strain) and \#41 (mutant strain) were performed as described [24]. Genomic DNA was further purified using Macherey-Nagel NucleoBond Xtra columns. The Illumina Paired-End sequencing was performed by ServiceXS using Illumina kits (cat\# 1001809 and 1005063) and protocols according to the instructions by the supplier. The quality and yield after sample preparation were checked and were consistent with the expected size of $300 \mathrm{bp}$ after excision from gel. Clustering and DNA sequencing using Illumina cBot and HiSeq 2000 was performed according to manufacturer's protocols. A total of 6.5 pmol of DNA was used. Two sequencing reads of 100 cycles each using Read1 sequencing and Read2 sequencing primers were performed with the flow cell. For strains 6.47 and \#41, 3.1 and 3.3 Gb of DNA sequence was obtained respectively. The genome sequences are available on request. Image analysis, base calling and quality check was performed with the Illumina data analysis pipeline RTA v1.13.48 and/or OLB v1.9/CASAVA v1.8.2. SNPs between the two strains (mutant \#41 and parental strain 6.47), were identified using $A$. niger strain ATCC1015 (http://genome. jgi-psf.org/pages/search-for-genes.jsf?organism=Aspni5) as a reference genome. Low quality bases were removed from the raw sequencing data. A Q25 phred score was used as a minimum and bases with phred scores below were removed and reads containing these bases were split. If the resulting reads from splits were shorter than 40 bases they were removed altogether. Alignment of filtered reads was performed with BWA (version 0.5.9) which lays the foundation for finding SNPs. For each SNP it was verified whether the SNP was in a predicted protein encoding region using the $A$. niger 3.0 genome at JGI using the SNP coordinates.

\section{Availability of supporting data}

The data set(s) supporting the results of this article are included within the article and its additional files. Genome sequences of RD6.47 and mutant \#41 are available upon request.

\section{Additional files}

\begin{abstract}
Additional file 1: Tabel S1. List of all SNPs identified in mutant \#41.
Additional file 2: Tabel S2. SNPs in coding regions in \#41.

Additional file 3: Figure S1. The Asn to Asp mutation at position 191 in mutant \#41 probably leads to misorientation of the substrate-orientation YFN-domain in UgeA. A) Protein alignment of the UgeA homologues from A. niger, A. nidulans, human and E. coli. Residues interacting with carbohydrate substrate are indicated with a black star, residues interacting with NAD substrate are indicated with a yellow star, the mutation identified in mutant \#41 (N191D) is indicated with the blue arrow. B) Cartoon representation of the crystal structure from $A$. nidulans (PDB ID: 4LIS, [22], with $\beta$ - strands 6 and 7 in red, residues interacting with carbohydrate substrate in black $C$ ) Space-filling model of UgeA; showing that the key enzymatic residues are located on the inside of UgeA D) Space-filling model of selected amino acid residues of UgeA: N191 in blue, residues forming $\beta$-strands 6 and 7 in red, residues interacting with carbohydrate substrate in black.
\end{abstract}

\section{Competing interest}

The authors declare that they have no competing interests.

\section{Authors' contribution}

JP, BT and EL carried out the immunological analyses, JP and MA carried out the molecular genetic studies, JP, BT, ID, CH and AR designed the initial experiments. All authors contributed to data interpretation and writing. All authors read and approved the final manuscript.

\section{Acknowledgements}

We thank Denise van Haren and Esther Goedkoop for their help in carrying out experiments on the complementation and genome analysis and Peter Eijsler for constructing the ugeA deletion strain. We thank Frank Ebel for the L10 antibody and Annika Pettersson and Dorien van 't Oever for the Platelia kits. This work was financially supported by the Dutch Technology Foundation (STW).

\section{Author details}

${ }^{1}$ Institute of Biology Leiden, Molecular Microbiology and Biotechnology, Leiden University, Sylviusweg 72, 2333, BE, Leiden, The Netherlands.

2Department of Molecular Cell Biology and Immunology, VU University 
Medical Center, van den Boechorststraat 7, 1081, BT, Amsterdam, The Netherlands. ${ }^{3}$ Current address: Department of Biological Sciences, Xi'an Jiaotong Liverpool University, 111 Ren Ai Road, Dushu Lake Higher Education Town, Suzhou Industrial Park, Suzhou, Jiangsu 215123, China.

Received: 21 August 2014 Accepted: 1 September 2014 Published online: 14 October 2014

\section{References}

1. Bernard M, Latgé JP: Aspergillus fumigatus cell wall: composition and biosynthesis. Med Mycol 2001, 39(Suppl 1):9-17.

2. Gastebois A, Clavaud C, Aimanianda V, Latgé J-P: Aspergillus fumigatus: cell wall polysaccharides, their biosynthesis and organization. Future Microbiol 2009, 4:583-595.

3. Klis FM, Ram AFJ, de Groot PWJ: A Molecular and Genomic View of the Fungal Cell Wall. In The Mycota VIII Biology of the Fungal Cell. Edited by Howard RJ, Gow NAR. Berlin: Springer-Verlag; 2007:97-120.

4. Tefsen B, Ram AFJ, van Die I, Routier FH: Galactofuranose in eukaryotes: aspects of biosynthesis and functional impact. Glycobiology 2012, 22:456-69.

5. Engel J, Schmalhorst PS, Dörk-Bousset T, Ferrières V, Routier FH: A single UDP-galactofuranose transporter is required for galactofuranosylation in Aspergillus fumigatus. J Biol Chem 2009, 284:33859-33868.

6. Afroz S, El-Ganiny AM, Sanders DAR, Kaminskyj SGW: Roles of the Aspergillus nidulans UDP-galactofuranose transporter, UgtA in hyphal morphogenesis, cell wall architecture, conidiation, and drug sensitivity. Fungal Genet Biol 2011, 48:896-903.

7. Komachi $Y$, Hatakeyama S, Motomatsu H, Futagami T, Kizjakina K, Sobrado $P$, Ekino K, Takegawa K, Goto M, Nomura Y, Oka T: GfsA encodes a novel galactofuranosyltransferase involved in biosynthesis of galactofuranose antigen of O-glycan in Aspergillus nidulans and Aspergillus fumigatus. Mol Microbiol 2013, 90:1054-1073.

8. Damveld RA, Franken A, Arentshorst M, Punt PJ, Klis FM, van den Hondel CA, Ram AF: A novel screening method for cell wall mutants in Aspergillus niger identifies UDP-galactopyranose mutase as an important protein in fungal cell wall biosynthesis. Genetics 2008, 178:873-881.

9. Schmalhorst PS, Krappmann S, Vervecken W, Rohde M, Müller M, Braus GH Contreras R, Braun A, Bakker H, Routier FH: Contribution of galactofuranose to the virulence of the opportunistic pathogen Aspergillus fumigatus. Eukaryot Cell 2008, 7:1268-1277.

10. El-Ganiny AM, Sanders DAR, Kaminskyj SGW: Aspergillus nidulans UDP-galactopyranose mutase, encoded by ugmA plays key roles in colony growth, hyphal morphogenesis, and conidiation. Fungal Genet Biol 2008, 45:1533-11542.

11. Heiss C, Skowyra ML, Liu H, Klutts JS, Wang Z, Williams M, Srikanta D, Beverley SM, Azadi P, Doering TL: Unusual galactofuranose modification of a capsule polysaccharide in the pathogenic yeast Cryptococcus neoformans. J Biol Chem 2013, 288:10994-11003.

12. Lee MJ, Gravelat FN, Cerone RP, Baptista SD, Campoli PV, Choe SI, Kravtsov I, Vinogradov E, Creuzenet C, Liu H, Berghuis AM, Latgé JP, Filler SG, Fontaine T, Sheppard DC: Overlapping and distinct roles of Aspergillus fumigatus UDP-glucose 4-epimerases in galactose metabolism and the synthesis of galactose-containing cell wall polysaccharides. J Biol Chem 2014, 289:1243-1256.

13. El-Ganiny AM, Sheoran I, Sanders DAR, Kaminskyj SGW: Aspergillus nidulans UDP-glucose-4-epimerase UgeA has multiple roles in wall architecture, hyphal morphogenesis, and asexual development. Fungal Genet Biol 2010, 47:629-635.

14. Heesemann L, Kotz A, Echtenacher B, Broniszewska M, Routier F, Hoffmann P, Ebel F: Studies on galactofuranose-containing glycostructures of the pathogenic mold Aspergillus fumigatus. Int J Med Microbiol 2011, 301:523-530.

15. Stynen D, Goris A, Sarfati J, Latgé JP: A new sensitive sandwich enzyme-linked immunosorbent assay to detect galactofuran in patients with invasive aspergillosis. J Clin Microbiol 1995, 33:497-500.

16. Bardalaye $\mathrm{PC}$, Nordin $\mathrm{JH}$ : Chemical structure of the galactomannan from the cell wall of Aspergillus niger. J Biol Chem 1977, 252:2584-2591.

17. Punt PJ, Schuren FHJ, Lehmbeck J, Christensen T, Hjort C, van den Hondel CAMJJ: Characterization of the Aspergillus niger PrtT, a unique regulator of extracellular protease encoding genes. Fungal Genet Biol 2008, 45:1591-1599.

18. Carvalho NDSP, Arentshorst M, Jin Kwon M, Meyer V, Ram AFJ: Expanding the ku70 toolbox for filamentous fungi: establishment of complementation vectors and recipient strains for advanced gene analyses. Appl Microbiol Biotechnol 2010, 87:1463-1473.

19. Gruben BS, Zhou M, de Vries RP: GalX regulates the D-galactose oxidoreductive pathway in Aspergillus niger. FEBS Lett 2012, 586:3980-3985.

20. Fekete E, de Vries RP, Seiboth B, vanKuyk PA, Sándor E, Fekete E, Metz B, Kubicek CP, Karaffa L: D-Galactose uptake is nonfunctional in the conidiospores of Aspergillus niger. FEMS Microbiol Lett 2012, 329:198-203.

21. Hayer K, Stratford M, Archer DB: Structural features of sugars that trigger or support conidial germination in the filamentous fungus Aspergillus niger. Appl Environ Microbiol 2013, 79:6924-6931.

22. Dalrymple SA, Ko J, Sheoran I, Kaminskyj SG, Sanders DA: Elucidation of substrate specificity in Aspergillus nidulans UDP-galactose-4-epimerase. PLoS One 2013, 8:e76803.

23. Bennett JW, Lasure LL: In More Gene Manipulations in Fungi. San Diego: Academic Press; 1991:441-447.

24. Arentshorst M, Ram AF, Meyer V: Using non-homologous end-joining-deficient strains for functional gene analyses in filamentous fungi. Methods Mol Biol 2012, 835:133-150

25. Meyer V, Arentshorst M, El-Ghezal A, Drews AC, Kooistra R, van den Hondel CA, Ram AF: Highly efficient gene targeting in the Aspergillus niger kusA mutant. J Biotechnol 2007, 128:770-775

26. Salvador LD, Suganuma T, Kitahara K, Tanoue H, Ichiki M: Monosaccharide composition of sweetpotato fiber and cell wall polysaccharides from sweetpotato, cassava, and potato analyzed by the high-performance anion exchange chromatography with pulsed amperometric detection method. J Agric Food Chem 2000, 48:3448-3454.

27. Carvalho ND, Arentshorst M, Jin Kwon M, Meyer V, Ram AF: Effects of a defective ERAD pathway on growth and heterologous protein production in Aspergillus niger. Appl Microbiol Biotechnol 2011, 89:357-373.

28. Kwon MJ, Arentshorst M, Fiedler M, de Groen FL, Punt PJ, Meyer V, Ram AF: Molecular genetic analysis of vesicular transport in Aspergillus niger reveals partial conservation of the molecular mechanism of exocytosis in fungi. Microbiology 2014, 160:316-329.

doi:10.1186/s40694-014-0006-7

Cite this article as: Park et al.: Identification of the UDP-glucose-4epimerase required for galactofuranose biosynthesis and galactose metabolism in A. niger. Fungal Biology and Biotechnology 2014 1:6.

\section{Submit your next manuscript to BioMed Central and take full advantage of:}

- Convenient online submission

- Thorough peer review

- No space constraints or color figure charges

- Immediate publication on acceptance

- Inclusion in PubMed, CAS, Scopus and Google Scholar

- Research which is freely available for redistribution

Submit your manuscript at www.biomedcentral.com/submit
C) Biomed Central 